\title{
Evaluation of Docking Calculations on X-Ray Structures Using CONSENSUS-DOCK
}

\author{
Masako Okamoto,* Yoshiaki Masuda, Ayumu Muroya, Kazuhiro Yasuno, Osamu Takahashi, and \\ Toshio FuruYA \\ Drug Discovery Department, Research \& Development Division, PharmaDesign, Inc.; 2-19-8 Hatchobori, Chuo-ku, \\ Tokyo 104-0032, Japan. Received July 23, 2010; accepted September 24, 2010; published online September 29, 2010
}

\begin{abstract}
We are participating in the challenge of identifying active compounds for target proteins using structurebased virtual screening (SBVS). We use an in-house customized docking program, CONSENSUS-DOCK, which is a customized version of the DOCK4 program in which three scoring functions (DOCK4, FlexX and PMF) and consensus scoring have been implemented. This paper compares the docking calculation results obtained using CONSENSUS-DOCK and DOCK4, and demonstrates that CONSENSUS-DOCK produces better results than DOCK4 for major X-ray structures obtained from the Protein Data Bank (PDB).
\end{abstract}

Key words structure-based virtual screening; CONSENSUS-DOCK; DOCK4

During the past decade, in silico approaches for seeking active compounds to target proteins have become more popular in drug discovery due to significant advances in computer hardware and software. There are a large number of docking programs (e.g., DOCK, ${ }^{1)}$ AutoDock, ${ }^{2)}$ GOLD, ${ }^{3)}$ GLIDE, ${ }^{4)} \mathrm{Ph}-$ $\mathrm{DOCK}^{5)}$ ), and many applications of these programs have been reported by various research groups. In major pharmaceutical companies, in silico approaches have been used to solve complicated drug design problems, leading to an increased appreciation and understanding of in silico approaches among medicinal chemists, pharmacological scientists and other researchers. ${ }^{6,7)}$

We have applied several in silico approaches to advance our drug discovery efforts, including structure-based virtual screening (SBVS), ligand-based drug design (LBDD), fragment-based drug design (FBDD), and assessment of proteinprotein interactions (PPI). ${ }^{8}$ We have been particularly successful in efficiently finding active compounds for various target proteins (e.g., GPCR, kinase) by SBVS, and have generally used an in-house docking program, CONSENSUSDOCK ${ }^{9)}$ CONSENSUS-DOCK is a customized version of the DOCK4 program in which three scoring functions (DOCK4, Flex ${ }^{10)}$ and $\mathrm{PMF}^{11)}$ ) and consensus scoring have been implemented. We previously reported the advantages of CONSENSUS-DOCK over DOCK4 in SBVS for a target protein, death-associated protein kinases (DAPKs). ${ }^{9)}$ To confirm the advantages of CONSENSUS-DOCK by using other types of proteins, we compare the docking calculation results obtained using CONSENSUS-DOCK and DOCK4 for $16 \mathrm{X}$ ray crystal structures in this paper. We report that CONSENSUS-DOCK provided better docking calculations than DOCK4 for most of the $16 \mathrm{X}$-ray structures chosen from the Protein Data Bank (PDB). ${ }^{12)}$

In CONSENSUS-DOCK, three scoring functions (DOCK4, FlexX and PMF) are implemented in the DOCK4 program and consensus scoring ${ }^{13}$ is performed in the pose selection, and the compounds are ranked to reduce the weaknesses of each scoring function. DOCK4 is a force field based docking program developed by Kuntz et al. ${ }^{1)}$ and is used for automated molecular docking of flexible molecules. DOCK4 uses electrostatic and van der Waals interactions evaluated over a grid to calculate the binding energy of a docked conformation. FlexX is an empirical scoring function and is implemented in the SYBYL package. Standard parameters of FlexX's scoring function are used for iterative growing and subsequent scoring of docking poses. PMF is a knowledge-based scoring function that combines the accuracy of empirical scoring functions with the advantage of higher generality and therefore wider applicability.

Various kinds of consensus scores have been used by sev-
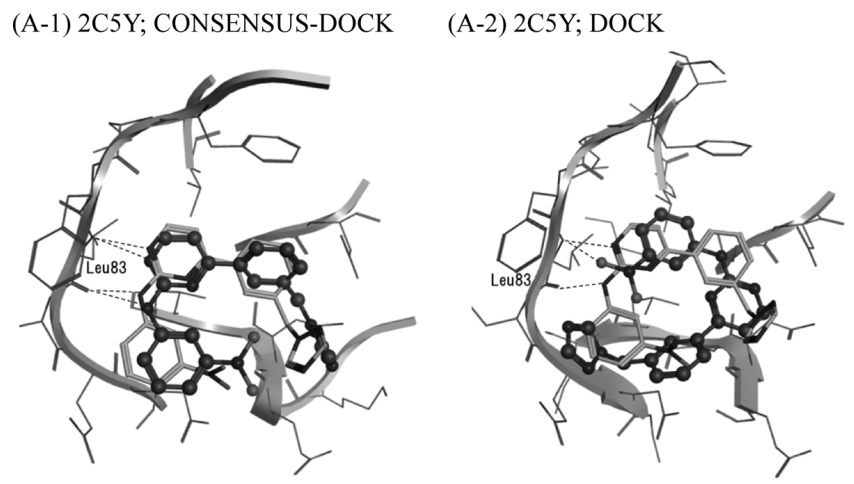

(B-1) 2A0C; CONSENSUS-DOCK

(B-2) $2 \mathrm{~A} 0 \mathrm{C}$; DOCK
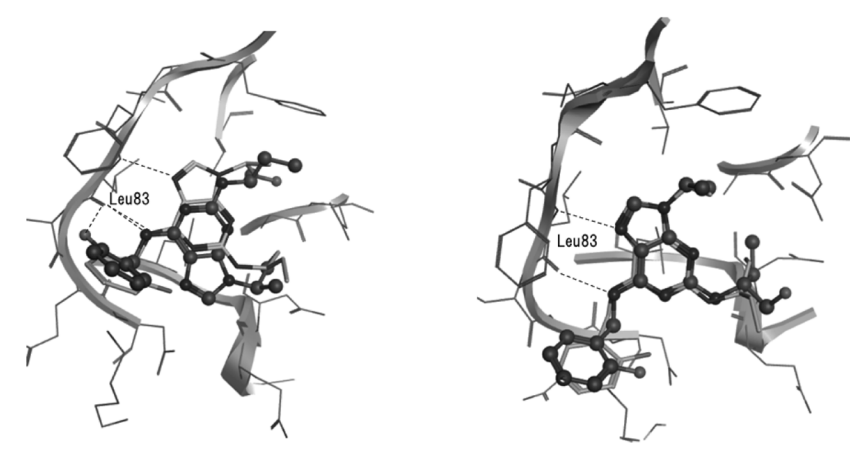

Fig. 1. Comparison of the Binding Modes

The X-ray complex structure is shown in light gray and the outputs of the docking calculations (DOCK, CONSENSUS-DOCK) are in dark gray. (A-1) (A-2) For 2C5Y, the output from CONSENSUS-DOCK was very similar to the X-ray structure. On the other hand, the output from DOCK was significantly different from the X-ray structure. (B-1) (B-2) For 2A0C, the output from DOCK was very similar to the X-ray structure, but the output from CONSENSUS-DOCK was incorrect. However, the difference was not radical, and was primarily due to the purine ring being flipped by the pyrimidine ring. 
eral research groups for virtual screening, ${ }^{14,15)}$ CONSENSUS-DOCK calculates these three scoring functions simultaneously. The consensus score of score 1 , score 2 and score 3 is defined by MAX (score 1, score 2, score 3), where MAX ( ) means the maximum value. In other words, we chose the most unfavorable value from the three scores. Using this definition of the consensus score, each scoring function complements the others by hiding artificial minima from each scoring function, and false positives in screening are decreased. This methodology has provided better virtual screening results than DOCK4.

Before SBVS, we usually examine the docking simulation by CONSENSUS-DOCK using a small-scale (approximately 1000 compounds) test-set database to determine the docking calculation conditions. These 1000 compounds are selected randomly from commercially available compound databases $^{16)}$ after filtering by druglikeness. ${ }^{17)}$ The test-set database includes active ligands (ligands in X-ray crystal complex structures are preferable). For each compound in the database, the molecules are washed (removal of salts or minor components, deprotonation of strong acids, and protonation of strong bases) by $\mathrm{MOE},{ }^{18}$ ) then the three-dimensional structure generator CORINA ${ }^{19}$ ) (version 3.46) was used to calculate three-dimensional structures, including stereoisomers and tautomers.

To compare docking calculation results from CONSENSUS-DOCK and DOCK4, we selected four types of proteins intensively researched by pharmaceutical companies and institutes as representative drug discovery targets: kinase, protease, nuclear receptor, and GPCR. One kinase, protease and nuclear receptor were selected: kinase; cyclin-dependent kinase 2 (CDK2), protease; Factor Xa, nuclear receptor; estrogen receptor (ER). For each protein, four X-ray crystal structures of the protein binding a different ligand was selected in order to examine their feature in the comparison of the docking calculations. In contrast, since there are only a few X-ray

Table 1. Selected X-Ray Crystal Complex Structures and Their PDB Code

(1)

crystal structures of GPCR, we used four related structures: beta2-adrenergic receptor (PDB code; 2RH1, 3D4S), beta1adrenergic receptor (2VT4), and A2A adenosine receptor (3EML). The selected X-ray complex structures are listed in Table 1.

Docking calculations were conducted using CONSENSUS-DOCK and DOCK4. In CONSENSUS-DOCK, scaling parameters were optimized for the equalization of the contributions of three functions (DOCK4, FlexX, and PMF). Note that in this paper, "DOCK4" refers to "the standard force field score of DOCK4."

The results are shown in Table 2 and Fig. 2. In Table 2, the ranking of each ligand from an X-ray crystal complex structure in the test-set database, and the root mean square deviation (RMSD) of the ligand docking calculation result against the ligand from the X-ray crystal complex structure, are shown together with the crystal structure PDB code. In Fig. 2 , the results are plotted using Spotfire ${ }^{20)}$ for easier comparison. For CDK2, CONSENSUS-DOCK showed better ranking results than DOCK4, but the CDK2 RMSDs were not as good as for the other three proteins, FXa, ER $\alpha$ and GPCR. These results are probably due to the compactness of the ligands and the large size of the ATP binding site, so several binding modes are likely. As shown in Fig. 1, there are a few cases in which the binding mode of the CONSENSUSDOCK output is partially flipped versus the X-ray structure, but overall the binding mode was not significantly different from the X-ray structure. Regarding FXa, CONSENSUS-

Table 2. Comparison of the Docking Calculation Results Obtained Using CONSENSUS-DOCK and DOCK4

\begin{tabular}{|c|c|c|c|c|}
\hline Protein & PDB code & Docking program & Ranking & RMSD \\
\hline \multirow[t]{8}{*}{ CDK2 } & 1B38 & CONSENSUS-DOCK & 1 & 2.344 \\
\hline & $2 \mathrm{~A} 0 \mathrm{C}$ & CONSENSUS-DOCK & 82 & 1.720 \\
\hline & $2 \mathrm{C} 5 \mathrm{Y}$ & CONSENSUS-DOCK & 20 & 1.442 \\
\hline & $2 \mathrm{R} 3 \mathrm{H}$ & CONSENSUS-DOCK & 193 & 2.599 \\
\hline & 1B38 & DOCK 4 & 1 & 3.582 \\
\hline & $2 \mathrm{~A} 0 \mathrm{C}$ & DOCK4 & 251 & 1.679 \\
\hline & $2 \mathrm{C} 5 \mathrm{Y}$ & DOCK4 & 301 & 6.567 \\
\hline & $2 \mathrm{R} 3 \mathrm{H}$ & DOCK4 & 548 & 6.096 \\
\hline \multirow[t]{8}{*}{$\mathrm{FXa}$} & $2 \mathrm{BOK}$ & CONSENSUS-DOCK & 2 & 0.711 \\
\hline & 2JKH & CONSENSUS-DOCK & 81 & 0.892 \\
\hline & $2 \mathrm{VWN}$ & CONSENSUS-DOCK & 346 & 2.505 \\
\hline & 2VWO & CONSENSUS-DOCK & 364 & 1.367 \\
\hline & 2BOK & DOCK 4 & 1 & 0.959 \\
\hline & 2JKH & DOCK4 & 67 & 0.749 \\
\hline & $2 \mathrm{VWN}$ & DOCK4 & 454 & 1.947 \\
\hline & 2VWO & DOCK4 & 482 & 1.396 \\
\hline \multirow[t]{8}{*}{$\operatorname{ER} \alpha$} & $1 \mathrm{GWR}$ & CONSENSUS-DOCK & 111 & 0.790 \\
\hline & 2P15 & CONSENSUS-DOCK & 30 & 0.988 \\
\hline & 2QZO & CONSENSUS-DOCK & 69 & 0.624 \\
\hline & 3ERT & CONSENSUS-DOCK & 1 & 0.786 \\
\hline & 1GWR & DOCK 4 & 295 & 0.790 \\
\hline & 2P15 & DOCK4 & 139 & 0.997 \\
\hline & 2QZO & DOCK4 & 120 & 1.649 \\
\hline & 3ERT & DOCK4 & 11 & 0.845 \\
\hline \multirow[t]{8}{*}{ GPCR } & 2RH1 & CONSENSUS-DOCK & 4 & 0.924 \\
\hline & 2VT4 & CONSENSUS-DOCK & 23 & 0.917 \\
\hline & $3 \mathrm{D} 4 \mathrm{~S}$ & CONSENSUS-DOCK & 89 & 1.167 \\
\hline & 3EML & CONSENSUS-DOCK & 640 & 2.12 \\
\hline & 2RH1 & DOCK4 & 16 & 0.998 \\
\hline & 2VT4 & DOCK4 & 155 & 0.895 \\
\hline & $3 \mathrm{D} 4 \mathrm{~S}$ & DOCK4 & 45 & 1.154 \\
\hline & 3EML & DOCK4 & 4 & 0.837 \\
\hline
\end{tabular}




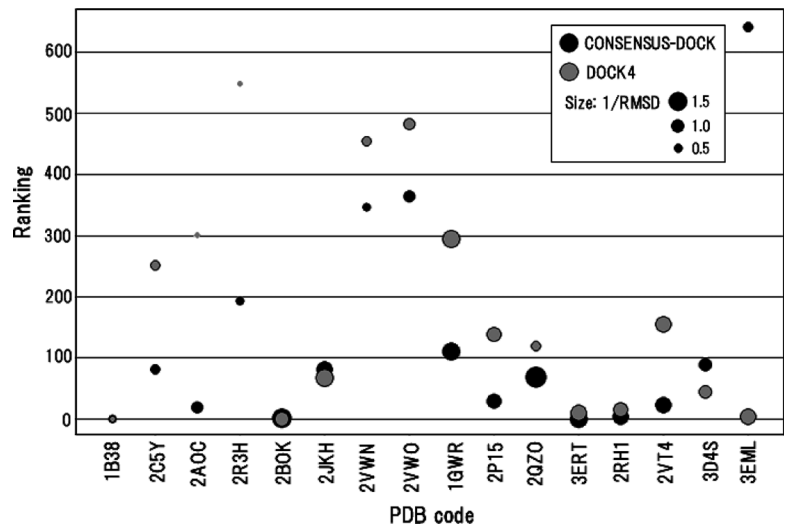

Fig. 2. Comparison of the Docking Calculation Results from CONSENSUS-DOCK and DOCK4

The dot color represents the docking program used: black; CONSENSUS-DOCK gray; DOCK4. The dot size reflects the reciprocal of the RMSD of the top ranking output from each docking calculation.

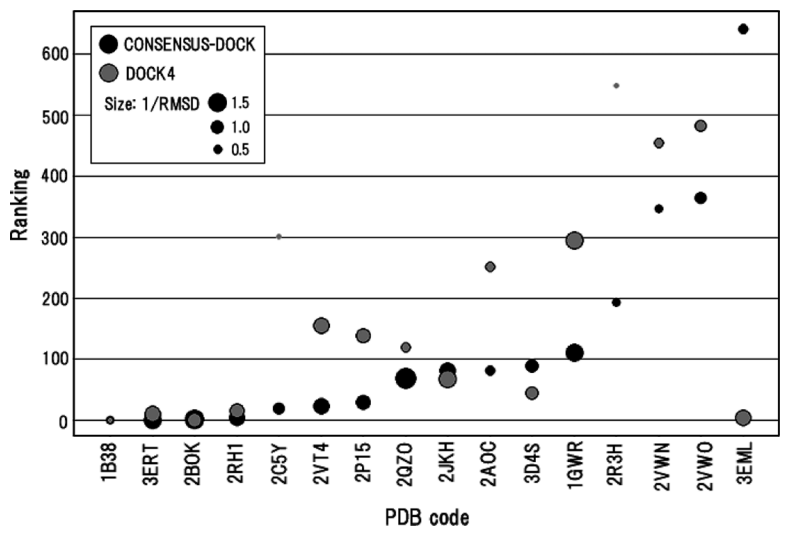

Fig. 3. RMSD Sorted by Ranking of CONSENSUS-DOCK

This graph clearly shows the advantage of CONSENSUS-DOCK over DOCK4. The dot color represents the docking program: black; CONSENSUS-DOCK, gray; DOCK4 The dot size reflects the reciprocal of the RMSD of the top ranking output from each docking calculation.

DOCK provided better results than DOCK4 for three of the four X-ray crystal structures; the exception was $2 \mathrm{JKH}$. Since the DOCK 4 score estimates the interaction energy between the ligand and the protein in the $2 \mathrm{JKH}$ complex better than the PMF score does, CONSENSUS-DOCK did not improve the ranking and the RMSD for this complex. For ER $\alpha, \mathrm{CON}$ SENSUS-DOCK provided better ranking and RMSD results than DOCK4 for all four crystal structures. For GPCR, CONSENSUS-DOCK provided better ranking results than DOCK4 for $2 \mathrm{RH} 1$ and $2 \mathrm{VT} 4$, but ranking results for $3 \mathrm{D} 4 \mathrm{~S}$ and 3EML were not good; in particular, the result for 3EML was much worse than the result obtained using DOCK4. This poor result came from the defectiveness of PMF to this target. When PMF was excluded from the scores used in CONSENSUS-DOCK, the ranking was improved.

The ranking results for the $16 \mathrm{X}$-ray complex structures calculated using CONSENSUS-DOCK or DOCK4 are shown in Fig. 3, and indicate that, in general, better results were obtained using CONSENSUS-DOCK. For most of the $\mathrm{X}$-ray structures, the RMSD results from CONSENSUSDOCK are better or similar to the results from DOCK4, and for 9 of the $16 \mathrm{X}$-ray structures, the ranking is significantly improved. Therefore, one of the merits of using CONSENSUS-DOCK appears to be its efficiency in ranking active

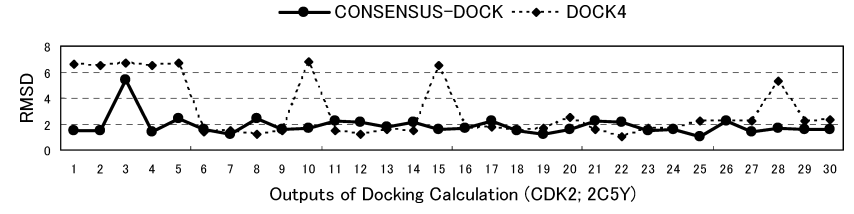

Fig. 4. Comparison of the RMSD of the Top 30 Outputs from Docking Calculations Using the CDK2 X-Ray Structure (2C5Y)

In CONSENSUS-DOCK, the outputs were sorted by CONSENSUS score, whereas in DOCK4, the outputs were sorted by DOCK4 docking score.

compounds in SBVS.

We also compared the RMSD of the outputs of the docking calculations (Fig. 4). We output 100 docking results about the ligand of CDK2 (PDB code; 2C5Y) and sorted by scoring. In CONSENSUS-DOCK, the outputs were sorted by CONSENSUS score, whereas in DOCK4, the outputs were sorted by DOCK4 implemented docking score. The top 30 outputs are shown in Fig. 4, the RMSD of the top two outputs in CONSENSUS-DOCK are below $1.5 \AA$. These results suggest that that CONSENSUS-DOCK provides a more accurate docking simulation than DOCK4.

In conclusion, we conducted docking simulations using CONSENSUS-DOCK and DOCK4 for 16 X-ray crystal complex structures. Comparison of the results demonstrated the advantage of CONSENSUS-DOCK over DOCK4 for a number of important X-ray complex structures. These results indicate the usefulness of CONSENSUS-DOCK in SBVS. We are in the process of applying SBVS protocols using CONSENSUS-DOCK to other medically important proteins, and will compare the results to other docking programs.

\section{References and Notes}

1) Ewing T. J., Kuntz I. D., J. Comput. Chem., 18, 1175-1189 (1997).

2) Morris G. M., Goodsell D. S., Halliday R. S., Huey R., Hart W. E., Belew R. K., Olson A. J., J. Comput. Chem., 19, 1639-1662 (1998).

3) Jones G., Willett P., Glen R. C., Leach A. R., Taylor R., J. Mol. Biol., 267, 727-748 (1997).

4) Friesner R. A., Banks J. L., Murphy R. B., Halgren T. A., Klicic J. J., Mainz D. T., Repasky M. P., Knoll E. H., Shelley M., Perry J. K., Shaw D. E., Fransis P., Shenkin P. S., J. Med. Chem., 47, 1739-1749 (2004).

5) Joseph-McCarthy D., Thomas B. E. I. V., Belmarsh M., Moustakas D., Alvarez J. C., Proteins: Struct., Funct., Bioinf., 51, 172-188 (2003).

6) Clark D. E., Expert Opin. Drug Discov., 3, 841—851 (2008).

7) Muegge I., Mini Rev. Med. Chem., 8, 927-933 (2008).

8) Sugaya N., Ikeda K., BMC Bioinformatics, 10, 263 (2009).

9) Okamoto M., Takayama K., Shimizu T., Ishida K., Takahashi O., Furuya T., J. Med. Chem., 52, 7323-7327 (2009).

10) Rarey M., Kramer B., Lengauer T., Klebe G., J. Mol. Biol., 261, 470489 (1996)

11) Muegge I., Martin Y. C., J. Med. Chem., 42, 791-804 (1999).

12) RCSB Protein Data Bank (http://www.rcsb.org/pdb/home/home.do).

13) Charifson P. S., Corkery J. J., Murcko M. A., Walters P., J. Med. Chem., 42, 5100-5109 (1999).

14) Park M.-S., Dessal A. L., Smrcka A. V., Stern H. A., J. Chem. Inf. Model., 49, 437-443 (2009).

15) Teramoto R., Fukunishi H., J. Chem. Inf. Model., 47, 526-534 (2007).

16) We used databases of commercially available compounds from several suppliers produced by Namiki Shoji Co., Ltd. (http://www.namikis.co.jp/).

17) Horio K., Muta H., Goto J., Hirayama N., Chem. Pharm. Bull., 55, 980-984 (2007).

18) Molecular Operating Environment (MOE 2008.1001), Chemical Computing Group Inc., 1010 Sherbrooke St. W, Suite 910, Montreal, Quebec, Canada H3A 2R7 (http://www.chemcomp.com/).

19) Sadowski J., Gasteiger J., Klebe G., J. Chem. Inf. Comput. Sci., 34 1000-1008 (1994). CORINA is available from Molecular Networks $\mathrm{GmbH}$, Erlangen, Germany (http://www.molecular-networks.com/)

20) TIBCO Spotfire 2.0 (http://spotfilre.tibco.jp) 